\title{
OBSERVATIONAL PHYSICS OF MIRROR WORLD
}

\author{
N.G. BOCHKAREV \\ Sternberg Astronomical Institute, Moscow, 119899, Russia \\ AND \\ M. YU. KHLOPOV \\ Scientific-Educational Center "Cosmion", Moscow, Russia
}

Mirror (shadow) particles are required to restore symmetry between left- and right-handed coordinate systems. If mirror world exists, it has been born at the same time as the ordinary world and has the same evolution (in the case of the shadow world - broken mirror symmetry, the evolution and structure of the shadow world does not correspond with the observed world). Mirror world is a kind of dark matter. According to Bahcall (1984) local dark matter has a density approximately equal to the density of observed (ordinary) matter.

Mirror (M) and ordinary ( 0 ) matter are two mutually penetrated worlds with the same sets of elementary particles in the same space; only gravitational (and possibly superweak) M-O interaction may exist. Astronomical observations is the only possible method for investigating M-world. M-matter via gravitational manifestations produces kinematical and gasdynamics effects. Island distribution of $\mathrm{O}-(\mathrm{M}-)$ barion excesses can lead to the existence of objects of definite mirrority with masses greater that those of globular clusters (up to clusters of galaxies). The lowest scale of Mobjects with observatinal manifestations corresponds to masses of planets. Visual manifestations are possible mainly on the scale $10^{10}-10^{20} \mathrm{~cm}$.

For scatchs of possible types of $\mathrm{M}$-worlds evidence see notes to Table 1 from Khlopov et al., 1991; see also Khlopov and Blinnikov, 1982.

\section{References}

Bahcall, J., 1984 ApJ, 286, 169; 273, 200

Blinnikov, S.N.and Khlopov, M.Yu. .Zh., 60, 632, 1982

Khlopov, M.Yu., Beskin, G.M., Bochkarev, N.G., Pustilnik, L.A., Pustilnik, S.A. 1991 Astron.Zh., 68, 42-57 (Sov.Astron. 35, 21-29) 